\title{
Structural Investigation of the Intermediate Layer in a Bimetal Mill Roller Produced by the Method of Vertical Axis Centrifugal Casting
}

\author{
Angel Velikov \\ Institute of Metal Science, Equipment \\ and Technologies with Centre of \\ Hydroaerodynamics \\ "Acad. A. Balevski" \\ Bulgarian Academy of Sciences \\ Sofia, Bulgaria \\ anmabg61@gmail.com
}

Abstract - Subject of the investigation is a bimetallic mill roller with external, working layer from high chromium material and inner, supporting layer from ductile cast iron. The processes, accompanying the formation of a transition layer in bimetallic castings, produced by centrifugal casting have been investigated. Attention has paid to the structural changes in the transition zone, caused by the diffusion processes occurring during the casting and crystallization.

Keywords - bimetal roller, centrifugal casting, mathematical model.

\section{INTRODUCTION}

The final structure of each cast material is a bearer the work properties of the casting. The requirements to the exploitation characteristics of castings type "roller" are that they must be with external work surface of high hardness and wear resistance, while the internal connecting, base surface must be of good workability and withstand heavy workloads. It is these requirements that lead to the idea that the casting must be made from two types of material: the external work surface of durable cast iron (in this case with high Cr content), and the inner one of ductile gray cast iron. Thus the machine processing is faced with some difficulties, so an additional requirement is set for the external surface to be in shape and dimensions as close as possible to those of a finished roller. This type of two-layer casting can be accomplished, applying the method of vertical axis centrifugal casting by successive pouring of the melts. For this purpose a special moulding equipment was constructed and made according to desired configuration of the casting. The values of the technological parameters for forming of the casting were

\author{
Ivan Georgiev \\ Institute of Metal Science, Equipment \\ and Technologies with Centre of \\ Hydroaerodynamics \\ "Acad. A. Balevski" \\ Bulgarian Academy of Sciences \\ Sofia, Bulgaria \\ ivan_georgiev51@abv.bg
}

determined, namely: the amount of the melts for the first and second layer, the mass rates of pouring the melts into the cavity of the metal mould, the type and thickness of the heat resistant [1] and at the same time thermally insulating coating on the working surfaces of the vertical shape, the pouring temperatures of the two types of melt, the angular velocity of the mould at forming of the casting, and the pause after the end of pouring of the first layer and the beginning of pouring of the second one.

A mathematical model of mould filling has been created. An experiment in real conditions was performed to determine the coefficient of heat transfer of the system "melt - refractory coating - metallic mould - environment".

From the experimental castings, metallographic specimens were cut, which were processed and prepared for structural testing.

The metallographic analysis was performed with an OPTON metallographic microscope.

\section{MATERIALS AND METHODS}

Technology requirements

The casting should be formed as follows:

1. First layer of alloy with high $\mathrm{Cr}$ content and chemical composition (table 1) with weight 1665kg;

2. Second layer of ductile cast iron with weight 2208 kg;

3. The two layers must be well soldered but not mixed and dissolved into each other. 
TABLE 1 FIRST LAYER OF ALLOY CHEMICAL COMPOSITION

\begin{tabular}{|c|c|c|c|c|c|}
\hline \multicolumn{7}{|c|}{ Chemical element, \% } \\
\hline $\boldsymbol{C}$ & $\boldsymbol{C r}$ & $\boldsymbol{S i}$ & $\mathbf{M n}$ & $\mathbf{N i}$ & $\mathbf{M o}$ \\
\hline $2,0 \div 3,2$ & $20 \div 25$ & $0,5 \div 1,0$ & $0,5 \div 1,5$ & $0,5 \div 1,5$ & $0,5 \div 2,0$ \\
\hline
\end{tabular}

Its geometry should be formed into a cylindrical mould represented schematically in Fig.1.

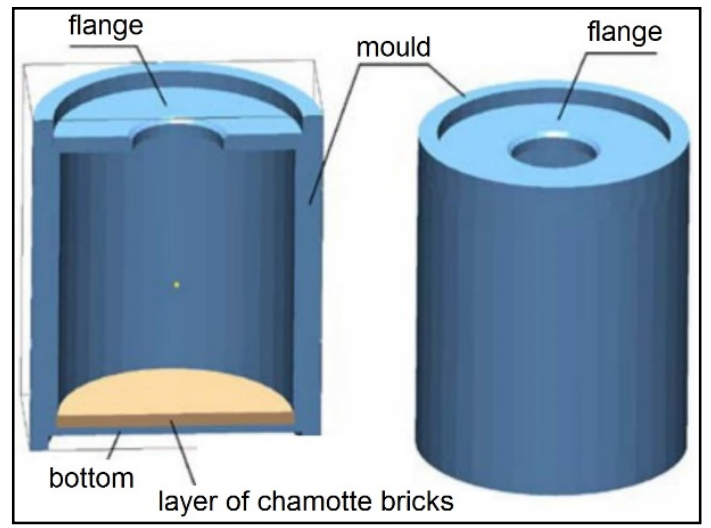

Fig. 1. Cylindrical mould

\section{RESULTS AND DISCUSSION}

A. Mathematical modelling of the geometry of the free surface of the fluid at rotation with constant angular velocity the mould.

The free surface equation is derived from the basic principle of the fluid's statics, which is that the resulting force is perpendicular to the free surface $[2,3,4]$. In the case of fluid with density $\rho$, rotating in a vessel with a vertical axis of rotation, the resultant force acting at a point of free surface and equal to the pressure is formed by the centrifugal force and the vertical component , balancing the weight of the fluid . These forces are shown schematically in Fig. 2.

For centrifugal force, written in components, we have:

$$
\vec{F}_{c}=\left(-\rho \omega^{2} r, 0\right)
$$

On the other hand:

$$
\vec{R}=-\vec{Q}=-(0,-\rho g)=(0, \rho g)
$$

The magnitude of the pressure $p$ is formed by the height of the fluid above the current point, i.e. $H-z(r)$ and therefore we will have:

$$
p=|\vec{p}|=p g[H-z(r)]
$$

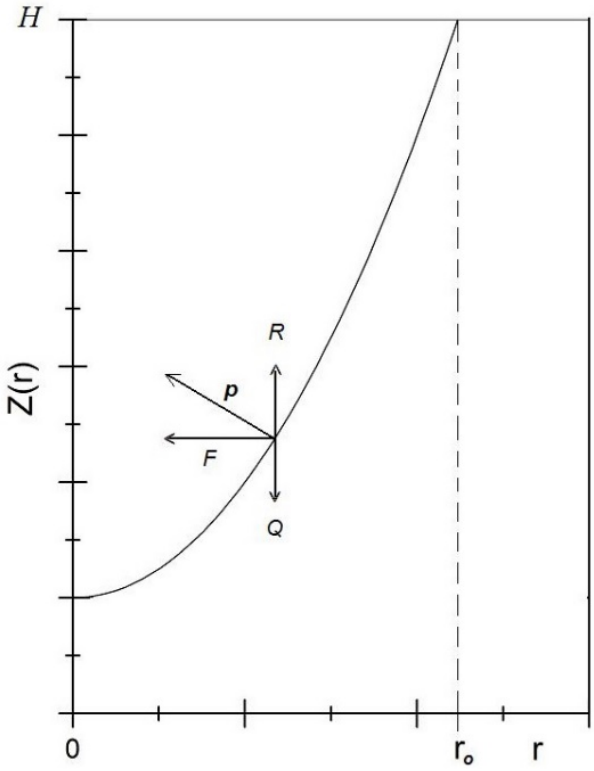

Fig. 2. Forces acting at a point of free surface

The normal single vector to the free surface can be found as follows:

First, we write the equation of the tangent to the curve of the surface at the current point. The equation turns out to be the following:

$$
y=z(r)+z^{\prime}(r)(x-r)
$$

where: $\boldsymbol{x}$ and $\boldsymbol{y}$ are the current coordinates of the tangent corresponding to $\boldsymbol{r}$ and $\boldsymbol{z}$. After an elementary transformation, the last equation turns out to be:

$$
-z^{\prime}(r) x+y-z(r)+r z^{\prime}(r)=0
$$

It is known from analytical geometry that the coefficients before $\boldsymbol{x}$ and $\boldsymbol{y}$ set a vector perpendicular to the line determined by equation $(3,5)$. Therefore the vector is collinear with $\vec{p}$ and perpendicular to the curve of surface. After its normalization of the type:

$$
\vec{e}_{n}=\frac{\left(-z^{\prime}(r), 1\right)}{\sqrt{1+z^{\prime 2}}}
$$

For $\vec{p}$ we get:

$$
\vec{p}=\frac{\rho g[H-Z(r)]}{\sqrt{1+z^{2}}}\left(-Z^{\prime}, 1\right)
$$

Further from the vector equation:

$$
\vec{p}=\vec{F}_{c}+\vec{R}
$$

Written in components, we get the following two differential equations: 


$$
\begin{aligned}
& \frac{g(H-z)}{\sqrt{1+z^{\prime}}} z^{\prime}=\omega^{2} r \\
& \frac{H-z}{\sqrt{1+z^{\prime 2}}}=1
\end{aligned}
$$

When substituting the second in the first it results in the following ordinary differential equation:

$$
\frac{d z(r)}{d r}=\frac{\omega^{2}}{g} r
$$

Its integration leads to the following solution:

$$
z(r)=\frac{\omega^{2}}{2 g} r^{2}+C
$$

determined to an accuracy of constant $\boldsymbol{C}$. It should be determined by the boundary condition:

$$
z\left(r_{0}\right)=H
$$

where: $\boldsymbol{r}_{\boldsymbol{o}}$ is the radius at which the maximum height of the vessel (mould) is reached. Then it turns out that $\boldsymbol{C}$ is:

$$
C=H-\frac{\omega^{2}}{2 g} r_{0}^{2}
$$

Substituting in (11) for the surface curve, we finally get:

$$
z(r)=H+\frac{\omega^{2}}{2 g}\left(r^{2}-r_{0}^{2}\right)
$$

It is apparent from this equation that the fluid surface takes the form of a rotary paraboloid. By the proper integration of the kind:

$$
V=2 \pi \int_{0}^{r_{0}} r z(r) d r
$$

the volume of fluid below the paraboloid can be calculated, i.e. the volume of fluid filling the cylindrical mould. With the mathematical model thus presented, solutions can be obtained for the free surface of a fluid under conditions of rotation at a constant angular velocity $\boldsymbol{\omega}$ and under the action of the earth's gravitational field, at different geometries of the mould, filled by the fluid and at different angular velocities.

\section{B. Calculating the pressure exerted of the liquid layer on the solidified layer.}

As a result of the active forces, a static pressure is formed at each point of the fluid, which changes both in radius and in height $[3,5]$. In this section, we will present a mathematical model that allows us to find this static pressure at any point in the fluid, and in particular on the wall of the mould or on an already solidified layer at formation of the casting at vertical axis centrifugal casting.
The static pressure in a point with coordinates $(\boldsymbol{r}, \mathbf{z})$ located in the liquid layer is formed by two components, as shown in Fig.3.

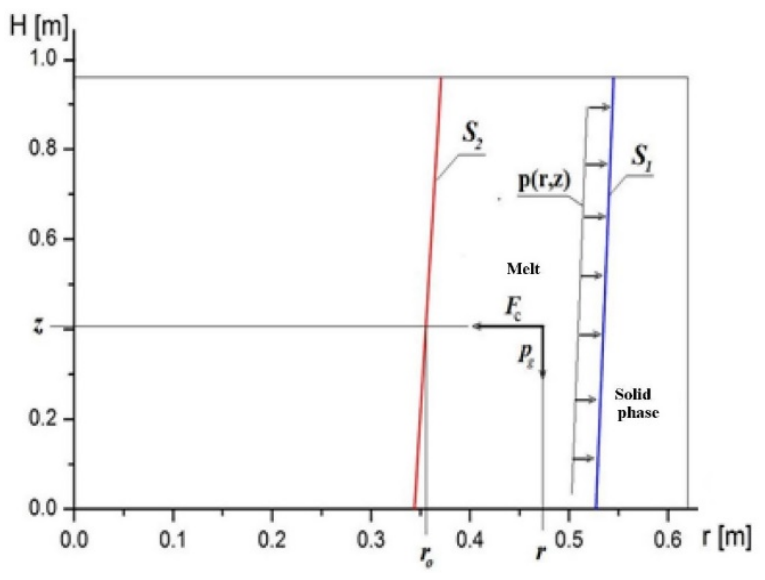

Fig. 3. Static pressure in a point with coordinates (r, z) located in the liquid layer

One is formed by the centrifugal force:

$$
F_{c}(r, z)=\int_{r_{0}}^{r} \rho \omega^{2} R d R=\frac{\rho \omega^{2}}{2}\left(r^{2}-r_{0}^{2}\right)
$$

and the other one by gravity:

$$
p_{m}=\rho g(H-z)
$$

where: $\boldsymbol{\rho}$ - is the mass density of the liquid layer; $\boldsymbol{\omega}$ angular velocity of rotation; $\boldsymbol{g}$ - earth acceleration.

Therefore, we can record the resultant pressure:

$$
p=\rho\left[g(H-z)+\frac{\rho \omega^{2}}{2}\left(r^{2}-r_{0}^{2}\right)\right]
$$

The last formula allows us to calculate the pressure at each point of the fluid. When $\boldsymbol{r}$ and $\mathbf{z}$ are coordinates of a point from the surface S1 of Fig. 3, we will obtain the pressure exerted of the fluid on the solid phase or mould.

Based on the mathematical model, a simulation was performed using the MAGMA Soft software package to determine the optimum casting temperatures of the first and second layers of the casting, as well as the required intermediate time between the completion of casting of the first layer and the start casting of the second layer, so that the two layers to be fused but not mixed.

\section{Structural observations of the formed intermediate layer.}

The specimen has been prepared in accordance with the standard methodology for metallographic analysis. Fig. 4 shows the cut-off specimen for metallographic observations. The research is focused on the so-called transition zone, between the external (working) layer of material with high Cr content and the inner (bearing) layer of ductile iron - Fig.5 to Fig.7. 
Angel Velikov, et al. Structural Investigation of the Intermediate Layer in a Bimetal Mill Roller Produced by the Method of Vertical Axis Centrifugal Casting

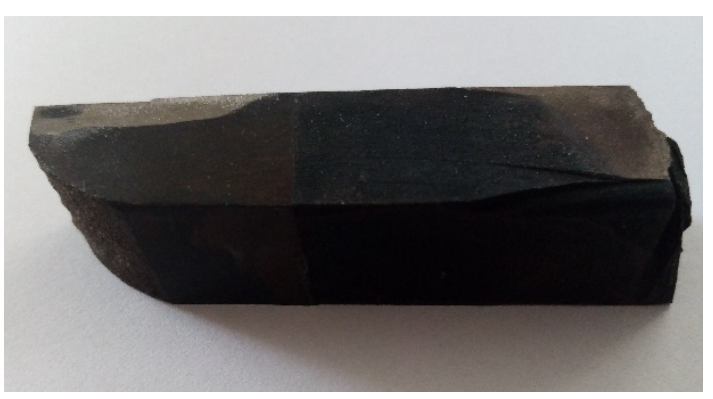

Fig. 4. Specimen for metallographic observations.

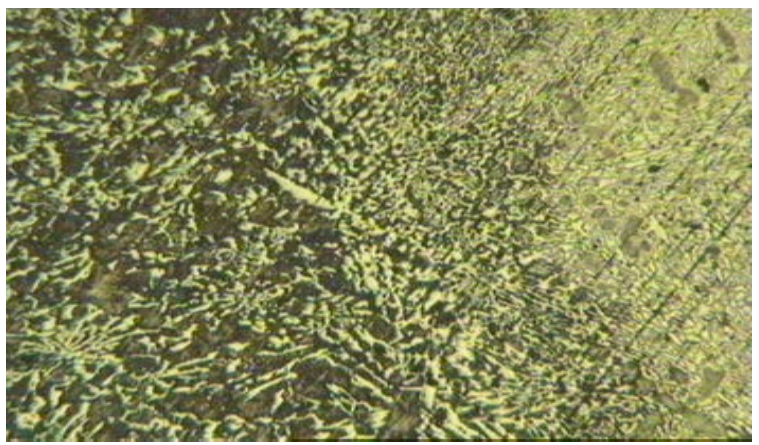

Fig. 5. Transition layer - x50

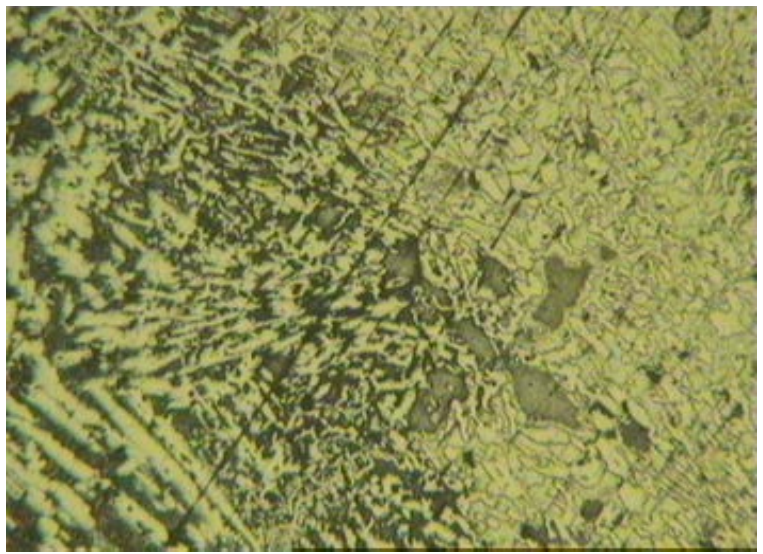

Fig. 6. Transition layer - x100

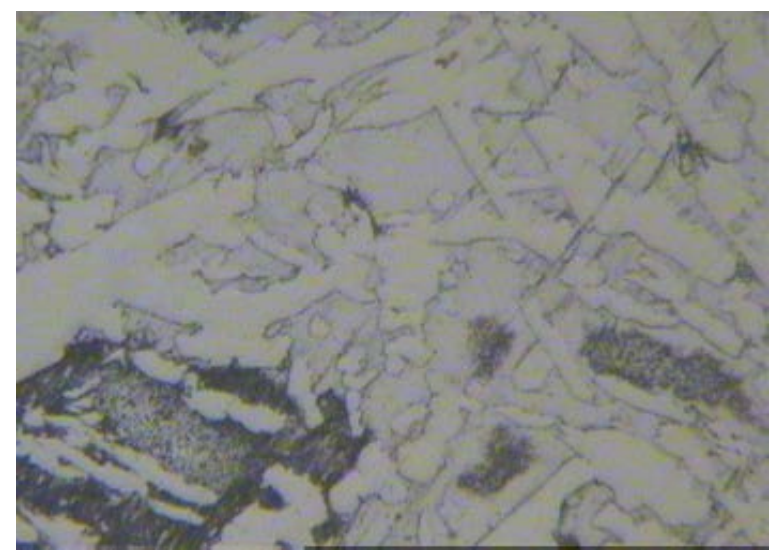

Fig. 7. Transition layer - x500

\section{CONCLUSIONS}

1. The metallographic analysis shows that the transition zone is possibly maximally limited.

2. The two materials are fully fused in the presence of minimal structural changes in the depth of the second (bearing) layer of ductile cast iron, caused by the diffusion processes accompanying the casting process of the inner layer and altering the initial chemical composition of the material.

\section{ACKNOWLEDGMENTS}

This work was supported by the European Regional Development Fund within the OP "Science and Education for Smart Growth 2014 - 2020", Project Co E "National center of mechatronics and clean technologies", № BG05M2OP001-1.001-0008-C08.

\section{REFERENCES}

[1] L.Gushev, V.Valkov and I.Georgiev "Modelling of the defining technological parameters in obtaining of heat-resistant coatings on molds for centrifugal casting”, Sat. Scientific Papers, vol. IX, pp. 177-187, Sept. 1986.

[2] G.Georgiev and I.Georgiev, "Mathematical modelling and computer simulation of two-component moulding”, Scientific Proceedings, year XXIII, vol. 2(165), June 2015.

[3] G. Georgiev, I. Georgiev, P.Ivanov, St. Tsenov and I.Panayotov, "Mathematical modelling and optimization of technology for the formation of two-layer casting by the method of centrifugal casting", XXIV International Scientific and Technical Conference : FOUNDRY 2017 proceedings, April 5-7, 2017, Pleven, Sofia, Scientific-technical union of mechanical engineering "Industry4.0”, 2018, pp23-28.

[4] S. Yudin, M. Levin, and S. Rosenfeld, Centrifugal Casting part 1, Moscow, 1972.

[5] I.Georgiev and V.Valkov, “Theoretical - experimental researches for determination of technological parameters at vertical axis centrifugal casting with of two-layer shaped castings”, Engineering Sciences, book 2, pp.86-94, Sept. 2011. 